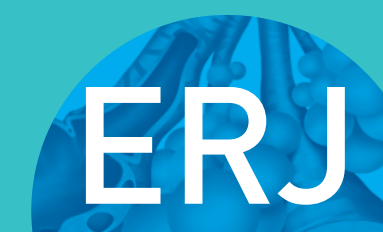

open research
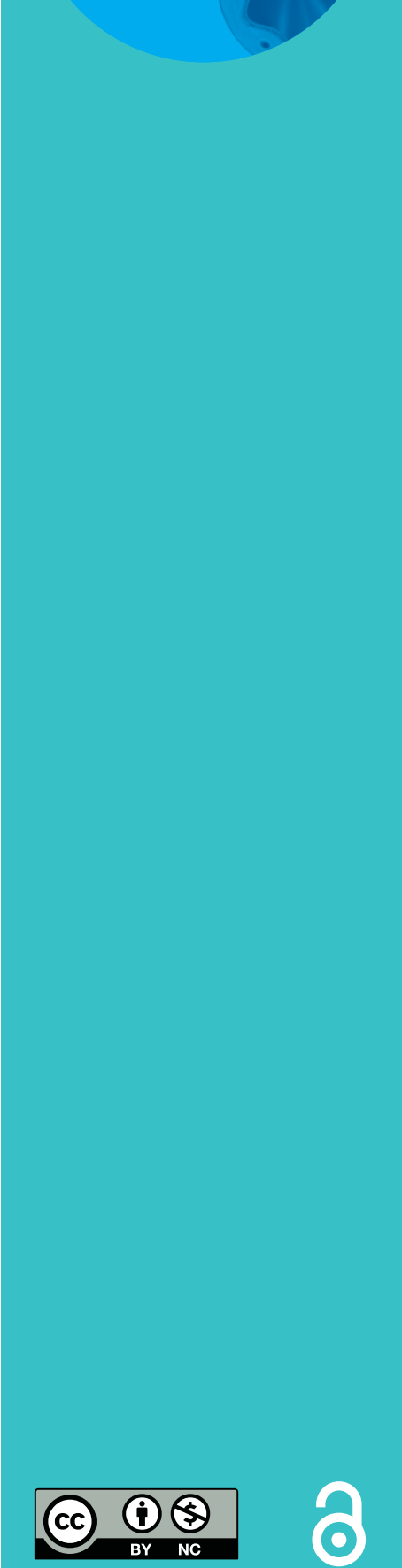

\section{Respiratory infection rates differ between geographically distant paediatric cystic fibrosis cohorts}

\author{
Kathryn A. Ramsey 1,2,7, Emily Hart ${ }^{3,4,7}$, Lidija Turkovic ${ }^{1}$, Marc Padros-Goossens ${ }^{1}$, \\ Stephen M. Stick ${ }^{1,5}$ and Sarath C. Ranganathan ${ }^{2,4,6}$ on behalf of the Australian \\ Respiratory Early Surveillance Team for Cystic Fibrosis (AREST CF) ${ }^{8}$
}

Affiliations: ${ }^{1}$ Telethon Kids Institute, University of Western Australia, Perth, Australia. ${ }^{2}$ Cystic Fibrosis Research and Treatment Center, University of North Carolina at Chapel Hill, Chapel Hill, NC, USA. ${ }^{3}$ Murdoch Children's Research Institute, Parkville, Australia. 'Dept of Respiratory Medicine, Royal Children's Hospital, Parkville, Australia. ${ }^{5}$ Dept of Respiratory Medicine, Princess Margaret Hospital for Children, Subiaco, Australia. ${ }^{6}$ Dept of Paediatrics, The University of Melbourne, Parkville, Australia. ${ }^{7}$ These authors contributed equally to this paper. ${ }^{8}$ The full membership of AREST CF is available at www.arestcf.org.

Correspondence: Kathryn Ramsey, Telethon Kids Institute, University of Western Australia, 100 Roberts Road, Subiaco, Western Australia 6008, Australia. E-mail: Kathryn.Ramseylatelethonkids.org.au

ABSTRACT Respiratory infections are a major cause of pulmonary decline in children with cystic fibrosis (CF). We compared the prevalence of infection in early life at geographically distant CF treatment centres participating in the same surveillance programme in Australia.

Lower airway microbiology, inflammation and structural lung disease at annual review were evaluated for 260 children 0-8 years old with CF at 1032 visits to CF treatment centres in Melbourne or Perth.

Melbourne patients were more likely to be culture-positive for common respiratory pathogens at all age groups (odds ratio (OR) 1.85, 95\% CI 1.33-2.58). Subjects <2 years old in Melbourne were also more likely to have neutrophil elastase present (OR 3.11,95\% CI 1.62-5.95). Bronchiectasis (OR 2.02, 95\% CI 1.21-3.38) and air trapping (OR 2.53, 95\% CI 1.42-4.51) in subjects 2-5 years old was more common in Melbourne subjects. The severity of structural lung disease was also worse in Melbourne patients $>5$ years old. Patients at both centres had a similar rate of hospitalisations and prescribed antibiotics.

No procedural differences were identified that could explain the disparity between pathogen prevalence. Geographical differences in early acquisition of infection may contribute to variability in outcomes between CF centres.

@ERSpublications

Infection rates different between two Australian paediatric cystic fibrosis patient cohorts http://ow.ly/PAmG302IYL4

This article has supplementary material available from openres.ersjournals.com

Received: Feb 022016 | Accepted after revision: July 262016

Support statement: Funding for the AREST CF programme was obtained from the Cystic Fibrosis Foundation Therapeutics (grants SLY04A0 and STICK09A0), and the National Health and Medical Research Council of Australia (NHMRC) (grants APP513730 and APP1020555, and Centre of Research Excellence \#1000896). K. Ramsey is an NHMRC Fellow (grant APP1088389). Funding information for this article has been deposited with the Open Funder Registry.

Conflict of interest: None declared.

The content of this work is copyright of the authors or their employers. Design and branding are copyright @ERS 2016. This article is open access and distributed under the terms of the Creative Commons Attribution Non-Commercial Licence 4.0. 


\section{Introduction}

Cystic fibrosis (CF) remains the most common life-limiting genetic condition occurring in Caucasian populations. In patients with $\mathrm{CF}$, progressive pulmonary damage occurs as a consequence of repeated airway infection and inflammation leading to respiratory failure. Lung disease starts early in life in individuals with $\mathrm{CF}$, characterised by abnormal lung function [1-3], pulmonary infection and inflammation [4-6], and structural lung abnormalities including bronchiectasis [7, 8]. While widespread introduction of newborn screening programmes has led to earlier diagnosis and improved nutritional status, treatment strategies aimed at halting disease progression during these formative years remain largely undefined.

The Australian Respiratory Early Surveillance Team for Cystic Fibrosis (AREST CF) was established to improve understanding of early CF lung disease and identify aspects of disease progression that may benefit from earlier intervention [7]. We now know that persistent structural changes occur within the lung much earlier than thought previously $[7,8]$, and are associated with pulmonary infection, worsening inflammation [9] and poorer nutritional status [10]. Inflammation has been linked to a reduction in infant lung function [2] and can be present either in the absence of pulmonary infection [11] or in association with specific infecting pathogens [12]. Infections have also been linked to reduced lung function throughout childhood [13]. Technological advances in the field of diagnostics have shown that microorganisms are present in the lower respiratory tract of children who are asymptomatic [7]. The epidemiology of respiratory tract infections in persons with CF has changed in recent years [14] and thus a clearer understanding of these pathogen populations will be imperative to directing the most suitable approaches to treatment.

In the present study we sought to monitor trends in pathogen prevalence by comparing two AREST CF patient cohorts attending treatment centres that are geographically distant, but which share similar approaches to treatment and use of antimicrobial prophylaxis. As we had shown previously that geographical differences occur in the first acquisition of infection with Pseudomonas aeruginosa at a single centre [15], we hypothesised that prevalence of infection would also be different at geographically distant CF centres.

\section{Methods}

Subjects

This study was a retrospective analysis of data collected as part of the AREST CF early surveillance programme. Infants and young children with CF were recruited to the AREST CF early surveillance programme from CF clinics in Melbourne (Royal Children's Hospital) and Perth (Princess Margaret Hospital for Children) in Australia. Participants underwent clinical assessment that included a chest computed tomography (CT) scan (at age 1, 3 and 5 years), bronchoscopy and bronchoalveolar lavage (BAL; performed annually from diagnosis up to 6 years old) for determination of pulmonary inflammation and infection. Research ethics committees at each of the participating institutions approved the early surveillance programme (Melbourne HREC25054; Perth 1762/EP) and consent of the parent/guardian of each patient was obtained. A detailed description of the methods is provided in the supplementary material.

\section{Data collection and analysis}

Bronchoscopy and BAL were performed under general anaesthesia, as described previously [5, 6], and the first recovered aliquot from each of the right middle lobe and lingula was sent to the central clinical laboratory for microbiological processing to detect bacterial, fungal and viral pathogens. Specimens were reported as culture-positive if there was growth of at least one predominant organism at a density $\geqslant 10^{4} \mathrm{CFU} \cdot \mathrm{mL}^{-1}$. Total cell counts were measured at each centre. Differential cell counts and inflammatory markers (interleukin 8 (IL-8) and neutrophil elastase (NE)) were assessed at the Perth centre as described previously [16]. The limit of detection for NE activity was $200 \mathrm{ng} \cdot \mathrm{mL}^{-1}$. For analytical purposes all samples with undetectable levels of NE were assigned an arbitrary value of $100 \mathrm{ng} \cdot \mathrm{mL}^{-1}$. Chest CT was performed at the time of the bronchoscopy. Scans were scored by an experienced paediatric thoracic radiologist at the Perth centre as described by Мотт et al. [9]. Demographics, mode of diagnosis, respiratory hospitalisations and CF transmembrane conductance regulator (CFTR) genotype were also recorded.

\section{Infection control and treatment}

Patients at both centres were seen at clinic every 3 months, and had equivalent infection control policies and treatment strategies in terms of prophylactic antibiotics, chronic medication use and airway clearance techniques. Both centres had a clinical policy to use anti-staphylococcal prophylaxis for a minimum of the first 2 years of life using amoxicillin/clavulanic acid to cover both Staphylococcus aureus and Haemophilus influenzae; however, adherence to this policy was not determined objectively. During exacerbations treatment with amoxicillin/clavulanic acid was usually maintained for at least 2 weeks before being changed to a second-line antibiotic if required. Perth and Melbourne AREST CF centres had an early detection and eradication programme for P. aeruginosa; once detected the child would receive 2 weeks of 
intravenous anti-pseudomonal antibiotics followed by 4 weeks of oral antibiotics and 1-2 months of inhaled tobramycin (supplementary table S1).

\section{Statistical analysis}

Differences in infection, inflammation and structural lung disease prevalence between centres were calculated using logistic regression models with infection, inflammation or structural lung disease presence as an outcome, with clustering for repeated observations on individuals. To assess the extent of structural lung disease, mixed-effect models with random intercepts were fitted to CT outcomes as a continuous variable, allowing for differences between individuals when they had repeated measures. Confounders were selected based on $\mathrm{p}<0.05$ in univariate analyses of outcomes and biologically plausible variables. To avoid collinearity only the stronger associated variable was included in the final model. All multivariate models were adjusted for age and pancreatic sufficiency. Presence and extent of inflammation and structural lung disease were also assessed separately in the age groups <2, 2-5 and $>5$ years. In all cases, model coefficients, 95\% confidence intervals and p-values were reported. Analyses were performed using Stata version 13.0 (Stata, College Station, TX, USA).

\section{Results}

\section{Patient demographics}

Microbiology and inflammatory data on 1037 BAL samples collected from 259 patients between January 2006 and December 2012 were included in this analysis. Data were available from 495 BAL samples collected from 111 Melbourne patients and 537 BAL samples from 149 Perth patients (table 1). Both cohorts were of a similar age; there were more males in Melbourne and more patients presenting by family history in the Melbourne group compared with Perth (table 1). Each centre tested a similar number of samples (on average 71 in Melbourne and 77 in Perth) from a similar number of patients (on average 64 in Melbourne and 72 in Perth) per year during the study period.

\section{Pathogen prevalence}

The Melbourne clinic reported more culture-positive samples per year: 56\% of Melbourne samples and $42 \%$ of Perth samples per year on average (odds ratio (OR) 1.85, 95\% CI 1.33-2.85; p<0.001), and recorded the presence of mixed oral flora more often than the clinic in Perth (67\% Melbourne, $45 \%$ Perth; OR 2.46, 95\% CI 1.86-3.25; p<0.001) (table 2). The majority of respiratory pathogens screened for were more prevalent among the Melbourne cohort, including S. aureus (OR 2.93, 95\% CI 1.67-5.15; p<0.001)

\section{TABLE 1 Study population}

\begin{tabular}{|c|c|c|}
\hline & Melbourne & Perth \\
\hline Subjects & 111 & 149 \\
\hline Visits & 495 & 537 \\
\hline Age at first BAL years & $0.3 \pm 0.1$ & $0.3 \pm 0.1$ \\
\hline Age at last BAL years & $6.0 \pm 0.1$ & $6.2 \pm 0.3$ \\
\hline Male & $66(59.6)$ & $67(45.2)$ \\
\hline$B M I$ at first $B A L \mathrm{~kg} \cdot \mathrm{m}^{-2}$ & $15.7 \pm 1.7$ & $15.3 \pm 2.3$ \\
\hline $\mathrm{BMI}$ at last $\mathrm{BAL} \mathrm{kg} \cdot \mathrm{m}^{-2}$ & $16.1 \pm 1.6$ & $15.7 \pm 1.7$ \\
\hline \multicolumn{3}{|l|}{ Mode of presentation } \\
\hline Newborn screening & $70(63.6)$ & 122 (85.9) \\
\hline Meconium ileus & $21(19.1)$ & $8(5.63)$ \\
\hline Family history & $8(7.3)$ & $1(0.7)$ \\
\hline Respiratory symptoms & $3(2.7)$ & $2(1.4)$ \\
\hline Failure to thrive & $1(0.9)$ & $6(4.2)$ \\
\hline Other & $7(6.4)$ & $3(2.1)$ \\
\hline \multicolumn{3}{|l|}{ Genotype } \\
\hline Homozygous Phe508del/Phe508del & $61(55.0)$ & $68(45.6)$ \\
\hline Heterozygous Phe508del & $39(35.1)$ & 72 (48.3) \\
\hline Other & $11(9.9)$ & $9(6.0)$ \\
\hline \multicolumn{3}{|l|}{ Hospitalisation and treatment } \\
\hline Patients with hospitalisation data & $66(59.4)$ & $66(44.3)$ \\
\hline Patients with no respiratory admissions & $75(67.4)$ & $96(64.0)$ \\
\hline Antibiotics prescribed & $105(94.1)$ & $131(87.9)$ \\
\hline
\end{tabular}




\begin{tabular}{|c|c|c|c|c|}
\hline & Melbourne & Perth & OR $(95 \% \mathrm{CI})$ & p-value \\
\hline Samples & 495 & 537 & & \\
\hline Any infection excluding mixed oral flora & $56.0(277)$ & $42.1(226)$ & $1.85(1.33-2.58)$ & $0.001^{\#}$ \\
\hline Aspergillus fumigatus & $9.5(47)$ & $9.1(49)$ & $1.05(0.61-1.82)$ & 0.85 \\
\hline Aspergillus species & $2.4(12)$ & $4.5(24)$ & $0.58(0.28-1.23)$ & 0.16 \\
\hline Candida albicans & $0.4(2)$ & $3.5(19)$ & $0.11(0.03-0.48)$ & $0.01^{\#}$ \\
\hline Escherichia coli & $4.2(21)$ & $0.7(4)$ & $5.09(0.96-26.96)$ & 0.06 \\
\hline Haemophilus influenzae & $15.6(77)$ & $6.3(34)$ & $3.00(1.80-5.01)$ & $0.001^{\#}$ \\
\hline Moraxella catarrhalis & $3.0(15)$ & $0.7(4)$ & $4.26(1.53-11.87)$ & $0.01^{\#}$ \\
\hline Mixed oral flora & $66.7(330)$ & $44.9(241)$ & $2.46(1.86-3.25)$ & $0.001^{\#}$ \\
\hline Pseudomonas aeruginosa & $8.3(41)$ & $10.8(58)$ & $0.75(0.43-1.30)$ & 0.30 \\
\hline Staphylococcus aureus & $18.4(91)$ & $8.0(43)$ & $2.93(1.67-5.15)$ & $0.001^{\#}$ \\
\hline
\end{tabular}

and $H$. influenzae (OR 3.00, 95\% CI 1.80-5.11; p<0.001) (table 2). Methicillin-resistant S. aureus was rarely reported in either group ( $0 \%$ Melbourne, $0.3 \%$ Perth) and the prevalence of $P$. aeruginosa infection was not difference between the two centres (OR 0.75, 95\% CI 0.43-1.30; p=0.30) (table 2). The higher prevalence of infection for pathogens screened in the Melbourne study centre compared with Perth remained nearly constant over time (figure 1).

\section{Treatment effects}

We next assessed treatment measures by comparing the annual number of respiratory admissions and antibiotic use that were entered in the national CF data registry for each of the patients included in the study. For both centres the majority of patients did not require hospitalisation for respiratory reasons (on average $67 \%$ of patients per year in Melbourne and $64 \%$ of patients per year in Perth) (table 1). The number of patients recorded to have prescribed antibiotics in any given year was also comparable (on average 94\% patients per year in Melbourne and 88\% patients per year in Perth; includes antimicrobial prophylaxis and treatment of respiratory exacerbations) (table 1).

\section{Disease severity}

To establish whether increased rates of infection were linked to poorer health outcomes, we then compared markers of pulmonary inflammation and measures of structural changes between patients attending either

FIGURE 1 Infection prevalence with age in Perth and Melbourne. Data is presented as proportion of children (0-1) at each age with a respiratory infection (pathogen density $\geqslant 10^{4} \mathrm{CFU} \cdot \mathrm{mL}^{-1}$ excluding mixed oral floral for Perth and Melbourne centres.

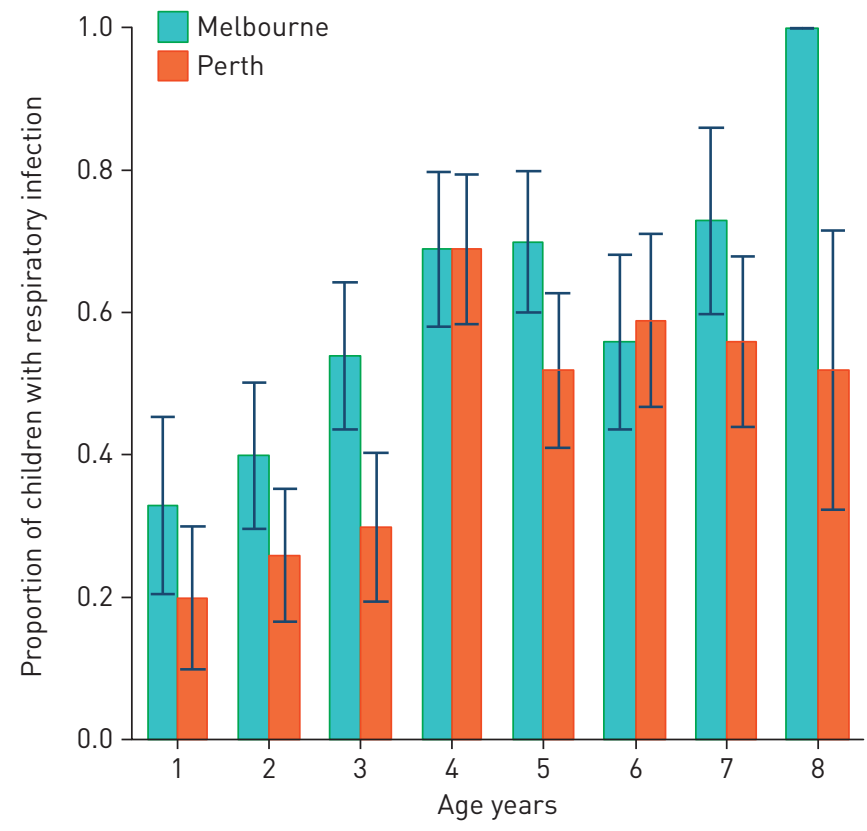


treatment centre. There was high variability in the measures obtained but overall, similar total cell counts and levels of IL-8 were observed for BAL taken from patients at both centres (table 3). Melbourne patients $<2$ years old were more likely to have detectable NE activity (OR 3.11, 95\% CI 1.62-5.95; p=0.001) (table 4). There was no significant difference between centres in the presence of IL-8 in any age group (table 4).

Comparison of structural disease revealed that the proportion of children with bronchiectasis and air trapping detected on chest CT was higher for Melbourne than Perth in patients 2-5 years old (OR 2.02, 95\% CI 1.21-3.38; $<<0.01$ and OR 2.53, 95\% CI 1.42-4.51; $<<0.01$, respectively) (table 4). In terms of extent, total CT damage, bronchiectasis and air trapping were more severe in the Melbourne cohort $>5$ years old when measured by volumetric scan on inspiration (table 5).

\section{Discussion}

In this study we found significant and consistent differences between the rates of lower respiratory tract infections reported at annual review for two geographically distant cohorts of young children with CF. This was despite both groups being treated similarly with regard to disease surveillance, infection control, protocols for procedures, treatment of exacerbations and use of antimicrobial prophylaxis. Although the pathogens most commonly identified at both centres were those traditionally associated with infections occurring during infancy and early childhood [17], the relative difference in frequency was surprising and was corroborated by findings of increased numbers of individuals with detectable lower respiratory free $\mathrm{NE}$ and increased presence and extent of structural lung disease.

Geographical variability in the prevalence of lower airway infection in patients with CF has been described previously. A comparison between adults attending British and American CF clinics revealed significant differences in the composition, abundance and diversity of the microorganisms present in the lungs of these two patient populations [18]. In a single-centre study we showed that children with CF residing in regional areas had an increased risk for first $P$. aeruginosa acquisition compared with their metropolitan-based counterparts [15]. In contrast, multicentre cohort studies conducted throughout Europe [19] and the USA [4] were unable to detect differences in the centre-specific prevalence of Burkholderia cepacia complex or common respiratory pathogens, respectively, due to the high diversity of species or strains among these pathogen populations. Notably, these studies focussed their investigations on either entire bacterial communities or a single pathogen, and generally used upper airway specimens to

TABLE 3 Inflammatory markers detected in bronchoalveolar lavage at annual review by age group

\begin{tabular}{|c|c|c|c|c|c|c|}
\hline & \multicolumn{2}{|c|}{$<2$ years } & \multicolumn{2}{|c|}{$2-5$ years } & \multicolumn{2}{|c|}{$>5$ years } \\
\hline Samples & 183 & 160 & 224 & 233 & 82 & 136 \\
\hline Total cell count $\times 10^{6} \mathrm{~mL}^{-1}$ & $1.75(0.68-3.42)$ & $1.57(0.85-2.49)$ & $3.95(2.25-6.63)$ & $3.72(2.42-5.67)$ & $4.75(2.33-8.13)$ & $4.31(2.56-7.52)$ \\
\hline IL- 8 concentration $\times 10^{2} \mathrm{pg} \cdot \mathrm{mL}^{-1}$ & $6.6(2.8-18.1)$ & $6.7(2.2-13.4)$ & $14.8(4.6-33.9)$ & $12.3(3.8-31.1)$ & $12.8(7.0-23.4)$ & $13.3(5.4-26.5)$ \\
\hline Detectable NE activity & $51 / 180(28)$ & $18 / 155$ (12) & $77 / 223$ (34) & $52 / 215(24)$ & 27/82 (35) & $52 / 128(41)$ \\
\hline $\mathrm{NE}$ concentration $\times 10^{2} \mathrm{ng} \cdot \mathrm{mL}^{-1}$ & $5.2(2.7-21.6)$ & $16.7(4.0-28.3)$ & $16.1(4.9-3.4)$ & $25.5(10.7-40.0)$ & $22.9(14.8-28.4)$ & $19.6(7.9-41.4)$ \\
\hline
\end{tabular}

TABLE 4 Difference in inflammatory markers detected in bronchoalveolar lavage and presence of structural lung disease detected by computed tomography scans by age group

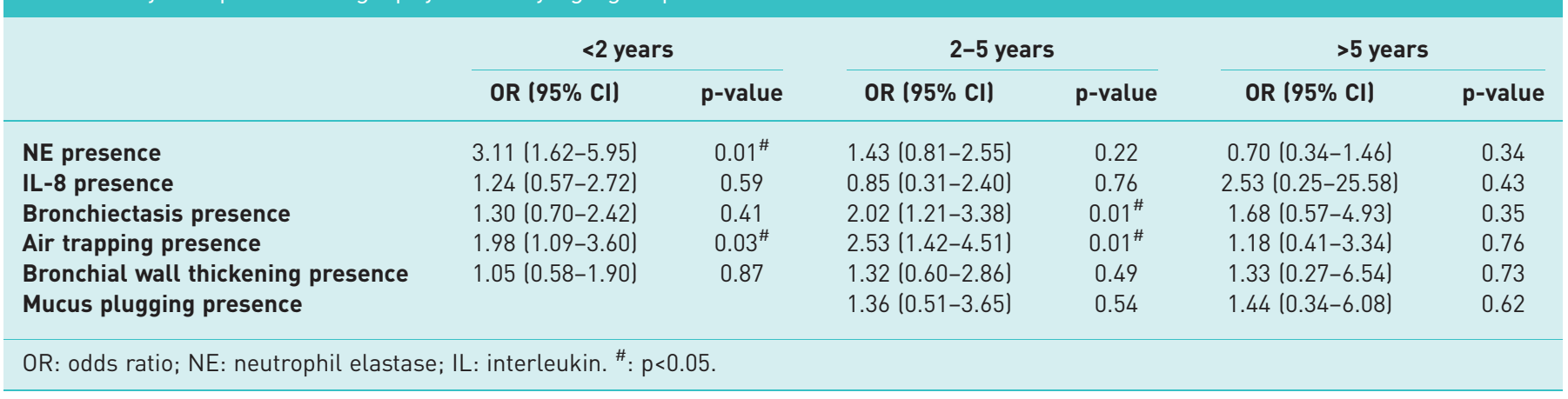


TABLE 5 Structural lung disease extent as detected by computed tomography (CT) scans at annual review by age group

\begin{tabular}{|c|c|c|c|c|c|c|}
\hline & \multicolumn{2}{|l|}{$<2$ years } & \multicolumn{2}{|c|}{$2-5$ years } & \multicolumn{2}{|c|}{$>5$ years } \\
\hline & OR $(95 \% \mathrm{CI})$ & p-value & OR $(95 \% \mathrm{CI})$ & p-value & OR $(95 \% \mathrm{CI})$ & p-value \\
\hline Total CT score & $-0.10(-1.58-1.38)$ & 0.89 & $1.73(-0.55-4.00)$ & 0.14 & $5.14(1.46-8.83)$ & $0.01^{\#}$ \\
\hline Air trapping score & $0.90(0.19-1.62)$ & $0.01^{\#}$ & $1.50(0.72-2.29)$ & 0.001 & $1.53(0.37-2.68)$ & $0.01^{\#}$ \\
\hline Bronchial wall thickening score & $-0.92(-1.82--0.01)$ & $0.04^{\#}$ & $-0.46(-1.50-0.58)$ & 0.39 & $1.14(-0.33-2.60)$ & 0.13 \\
\hline Mucus plugging score & $0.00(-0.10-0.09)$ & 0.93 & $-0.15(-0.46-0.17)$ & 0.36 & $0.42(-0.26-1.11)$ & 0.23 \\
\hline
\end{tabular}

study strain distribution. Our study is therefore one of the first reports to investigate region-specific prevalence of pathogens in the lower airways of similarly matched cohorts of young children with CF. The present study is unique in that it evaluated very young children at two centres united by a common disease surveillance programme and treatment protocols. Interestingly, these findings may contribute to explaining consistently better lung function for Perth subjects versus Melbourne subjects recorded in the national data registry [20], suggesting that these differences in disease outcomes may have their origins in part during the pre-school years.

In comparison with registry data, which record all exacerbation events, our surveillance cultures were performed once annually at a time when patients were relatively healthy. Although routine surveillance provides an opportunity to detect infection prior to the onset of symptoms, a limitation of this approach is the reduced likelihood of detecting species, such as Stenotrophomonas maltophilia and Alcaligenes xylosoxidans, that tend to only transiently infect the CF airways [21] and may explain why these organisms were rarely identified in surveillance cultures. Nevertheless, surveillance testing also allows the tracking of pathogen trends irrespective of exacerbation rates.

We did not take into account seasonality, which is known to influence acquisition of organisms such as $P$. aeruginosa [22], but this is unlikely to have explained centre differences in infections as surveillance BALs were performed throughout the year and not during exacerbations, which are more likely during the winter months. A study of the US and Australian CF registries found that higher ambient temperature was associated with earlier P. aeruginosa infection and lower lung function [23]. In addition, data from a large US study suggest that macro-environmental factors, such as temperature, humidity and air pollution, may contribute to the risk of $P$. aeruginosa acquisition [24]. It is possible that differences in macroenvironmental factors between Perth and Melbourne centres contributed to the difference in infection rates between the two centres.

The prevalence of $S$. aureus and $H$. influenzae infections has increased in recent years [25]. While this may reflect a change in strain prevalence or population exposure, such increases could also result from changes to diagnostic and/or reporting procedures. A detailed examination of $S$. aureus infections among this cohort revealed high diversity among the bacterial strain population and failed to identify any dominant clones that were specifically associated with respiratory infection in CF [26]. In addition, strain prevalence rates among the AREST CF cohort did not surpass those reported for the Australian CF population as a whole. As the aggressive use of antibiotics is a strong driver of changes to microbial community diversity, the emergence or expansion of specific species can often be attributed to alterations in the treatment being provided [27], but we were unable to detect such differences between centres, with the vast majority being prescribed amoxycillin/clavulanic acid as prophylaxis and a similar-sized minority being admitted for intravenous antibiotics to each centre or undergoing therapy to eradicate infection with $P$. aeruginosa.

For both centres involved in the AREST CF surveillance programme, early detection and eradication of $P$. aeruginosa infection has become routine practice, and it is clinical policy to use anti-staphylococcal prophylaxis for the first 2 years of life. That said, the prescription of the relevant antimicrobials is still dependent on preference of the treating physician and the willingness of parents to administer long-term treatment to well-looking children. Hence, subtle variations both within and between centres likely existed [10], but it is unclear whether these are substantial enough to explain such significant differences in the detection of lower respiratory infections treated by these antibiotics or the increased pulmonary inflammation and structural changes identified in the lungs of these very young subjects.

The prevalence of meconium ileus was higher in infants with CF from Melbourne (19\%) compared with Perth (6\%). It is still unclear whether individuals with meconium ileus at presentation have a worse prognosis 
than those without meconium ileus [28-30]. However, a previous study from the AREST CF group found that meconium ileus at presentation was a significant risk factor for the presence of bronchiectasis on chest $\mathrm{CT}$ at 3 years old [31]; therefore, centre differences in the prevalence of meconium ileus may have contributed to the observed differences in clinical status. We could not identify any procedural differences in sample collection or laboratory protocol that could explain our findings. Both the accredited laboratories meet national standards and are experienced laboratories for detection of CF infections. Our assessment of the administration of and adherence to treatment was, however, limited. An additional limitation of our study was that our analysis was restricted to the culture-based detection of a select number of respiratory pathogens and therefore did not take into account the entire lung microbiota that is currently under intense investigation utilising culture-independent techniques. Whether or not the nature of these complex polymicrobial communities influences disease progression is not yet known and so the presence and prevalence of organisms not studied here will be an important consideration for future investigations.

In addition to the detection of common respiratory pathogens, a large number of BAL specimens also grew mixed oral flora despite considerable care having been taken to avoid contamination from the upper airways. The significance of mixed oral flora to pulmonary disease in CF remains unclear. In a 3-year study investigating the ability of oropharyngeal cultures to predict infection within the lower airways most BAL cultures contained low numbers of organisms associated with the upper airways [32]. This was in contrast to earlier findings that BAL specimens did not contain mixed oral flora from a similar investigation comparing upper and lower CF airway microbiology [33]. Although not traditionally considered as pathogens, we have recently shown that the presence of mixed oral flora in the lower CF airways is associated with an increased inflammatory response [12]. While both centres aimed to record upper respiratory tract flora regardless of whether or not another pathogen was also reported, it is difficult to explain such disparity between the two groups without assuming some differences between diagnostic interpretation and reporting exist. However, having considered that variation in sample collection, processing and reporting may have occurred through employment of different bronchoscopists or diagnostic laboratories we reviewed all methodologies and were unable to identify any variation in procedures. Further research incorporating culture-independent techniques would assist in identifying what role, if any, mixed oral flora plays in early CF airway disease.

Although the extent of bronchiectasis was more severe among Melbourne patients, the use of two different scanners means that there may be subtle differences in image quality. However, the same investigator reviewed all CT scans, thus reducing the likelihood of differences in interpretation between centres. Volumetric chest CT scans provide a more comprehensive and detailed assessment of structural change within the airways than limited-slice imaging. Limited-slice protocols, which were used more in the Melbourne centre, are more likely to underestimate structural disease prevalence and extent, so it is unlikely that differences in scanning techniques accounted for these results.

In summary, we demonstrated that two CF treatment centres performing identical disease surveillance and utilising similar standardised treatment protocols had different prevalence of early respiratory infection. The centre with the higher infection rates also had a higher prevalence of free NE and worse chest CT findings. This study provides evidence for potential geographical variability in the acquisition of early lower respiratory infection in CF. Differences in rates of infection during the first few years of life may be associated with poorer disease outcomes, and warrant specific surveillance and intervention. Future, larger studies may identify that geographical differences in acquisition of infection contribute to the variability in outcomes that occurs between CF centres.

\section{Acknowledgements}

The authors acknowledge the assistance of Billy Skoric and Judy Park (Telethon Kids Institute, Subiaco, Australia), and thank the participants and their families who contribute to the AREST CF programme.

\section{References}

1 Lum S, Gustafsson P, Ljungberg $\mathrm{H}$, et al. Early detection of cystic fibrosis lung disease: multiple-breath washout versus raised volume tests. Thorax 2007; 62: 341-347.

2 Pillarisetti N, Williamson E, Linnane B, et al. Infection, inflammation, and lung function decline in infants with cystic fibrosis. Am J Respir Crit Care Med 2011; 184: 75-81.

3 Rosenfeld M, Farrell PM, Kloster M, et al. Association of lung function, chest radiographs and clinical features in infants with cystic fibrosis. Eur Respir J 2013; 42: 1545-1552.

4 Rosenfeld M, Gibson RL, McNamara S, et al. Early pulmonary infection, inflammation, and clinical outcomes in infants with cystic fibrosis. Pediatr Pulmonol 2001; 32: 356-366.

5 Armstrong DS, Hook SM, Jamsen KM, et al. Lower airway inflammation in infants with cystic fibrosis detected by newborn screening. Pediatr Pulmonol 2005; 40: 500-510.

6 Balough K, McCubbin M, Weinberger M, et al. The relationship between infection and inflammation in the early stages of lung disease from cystic fibrosis. Pediatr Pulmonol 1995; 20: 63-70.

7 Sly PD, Brennan S, Gangell C, et al. Lung disease at diagnosis in infants with cystic fibrosis detected by newborn screening. Am J Respir Crit Care Med 2009; 180: 146-152. 
Stick SM, Brennan S, Murray C, et al. Bronchiectasis in infants and preschool children diagnosed with cystic fibrosis after newborn screening. J Pediatr 2009; 155: 623-628.

9 Mott LS, Park J, Murray CP, et al. Progression of early structural lung disease in young children with cystic fibrosis assessed using CT. Thorax 2012; 67: 509-516.

10 Ranganathan SC, Parsons F, Gangell C, et al. Evolution of pulmonary inflammation and nutritional status in infants and young children with cystic fibrosis. Thorax 2011; 66: 408-413.

11 Brennan S, Sly PD, Gangell CL, et al. Alveolar macrophages and CC chemokines are increased in children with cystic fibrosis. Eur Respir J 2009; 34: 655-661.

12 Gangell C, Gard S, Douglas T, et al. Inflammatory responses to individual microorganisms in the lungs of children with cystic fibrosis. Clin Infect Dis 2011; 53: 425-432.

13 Ramsey KA, Ranganathan S, Park J, et al. Early respiratory infection is associated with reduced spirometry in children with cystic fibrosis. Am J Respir Crit Care Med 2014; 190: 1111-1116.

14 Lipuma JJ. The changing microbial epidemiology in cystic fibrosis. Clin Microbiol Rev 2010; 23: $299-323$.

15 Ranganathan SC, Skoric B, Ramsay KA, et al. Geographical differences in first acquisition of Pseudomonas aeruginosa in cystic fibrosis. Ann Am Thorac Soc 2013; 10: 108-114.

16 Brennan S, Hall GL, Horak F, et al. Correlation of forced oscillation technique in preschool children with cystic fibrosis with pulmonary inflammation. Thorax 2005; 60: 159-163.

17 Hauser AR, Jain M, Bar-Meir M, et al. Clinical significance of microbial infection and adaptation in cystic fibrosis Clin Microbiol Rev 2011; 24: 29-70.

18 Stressmann FA, Rogers GB, Klem ER, et al. Analysis of the bacterial communities present in lungs of patients with cystic fibrosis from American and British centers. J Clin Microbiol 2011; 49: 281-291.

19 McDowell A, Mahenthiralingam E, Dunbar KE, et al. Epidemiology of Burkholderia cepacia complex species recovered from cystic fibrosis patients: issues related to patient segregation. J Med Microbiol 2004; 53: 663-668.

20 Cystic Fibrosis Australia. Cystic Fibrosis in Australia 2007-2011. Annual Reports from the Australian Cystic Fibrosis Data Registry. www.cysticfibrosis.org.au/cfa/data-registry Date last accessed: December 14, 2012.

21 Demko CA, Stern RC, Doershuk CF. Stenotrophomonas maltophilia in cystic fibrosis: incidence and prevalence. Pediatr Pulmonol 1998; 25: 304-308.

22 Psoter KJ, De Roos AJ, Wakefield J, et al. Season is associated with Pseudomonas aeruginosa acquisition in young children with cystic fibrosis. Clin Microbiol Infect 2013; 19: 483-489.

23 Collaco JM, McGready J, Green DM, et al. Effect of temperature on cystic fibrosis lung disease and infections: a replicated cohort study. PLoS One 2011; 6: e27784.

24 Psoter KJ, Rosenfeld M, De Roos AJ, et al. Differential geographical risk of initial Pseudomonas aeruginosa acquisition in young US children with cystic fibrosis. Am I Epidemiol 2014; 179: 1503-1513.

25 Razvi S, Quittell L, Sewall A, et al. Respiratory microbiology of patients with cystic fibrosis in the United States, 1995 to 2005. Chest 2009; 136: 1554-1560.

26 Hart E, Wong J, Carzino R, et al. Molecular epidemiology of Staphylococcus aureus infections in a paediatric treatment centre. Am J Respir Crit Care Med 2012; 185: A5481.

27 Zhao J, Schloss PD, Kalikin LM, et al. Decade-long bacterial community dynamics in cystic fibrosis airways Proc Natl Acad Sci USA 2012; 109: 5809-5814.

28 Munck A, Gerardin M, Alberti C, et al. Clinical outcome of cystic fibrosis presenting with or without meconium ileus: a matched cohort study. J Pediatr Surg 2006; 41: 1556-1560.

29 Sanders DB, Li Z, Laxova A, et al. Risk factors for the progression of cystic fibrosis lung disease throughout childhood. Ann Am Thorac Soc 2014; 11: 63-72.

30 Evans AK, Fitzgerald DA, McKay KO. The impact of meconium ileus on the clinical course of children with cystic fibrosis. Eur Respir J 2001; 18: 784-789.

31 Sly PD, Gangell CL, Chen L, et al. Risk factors for bronchiectasis in children with cystic fibrosis. $N$ Engl J Med 2013; 368: 1963-1970.

32 Armstrong DS, Grimwood K, Carlin JB, et al. Bronchoalveolar lavage or oropharyngeal cultures to identify lower respiratory pathogens in infants with cystic fibrosis. Pediatr Pulmonol 1996; 21: 267-275.

33 Ramsey BW, Wentz KR, Smith AL, et al. Predictive value of oropharyngeal cultures for identifying lower airway bacteria in cystic fibrosis patients. Am Rev Respir Dis 1991; 144: 331-337. 